soft tissue. Prior to image analysis, an atlas of normal findings and measurements was comprised. Group differences were investigated using students T-test for continuous data and Fisher's exact test for nominal and ordinal data.

Results: A total of 40 patients were included in this retrospective casecontrol study, 20 in each group (RA and control). Position of dens was significantly more cranial in the RA group (dens to McGregor line: -1.62 $\mathrm{mm}$ for RA vs. $2.15 \mathrm{~mm}$ for controls, $p=0.002$; dens to McRae line: 3.20 for RA vs. 5.36 for controls, $\mathrm{p}<0.001)$. Visibility of alar ligaments was significantly lower in RA patients (fully visible in $10 \%$ of RA and $35 \%$ of control patients, $p=0.012$ ). Similarly, signal of transverse ligaments was normal in $5 \%$ of RA vs. $35 \%$ of control patients $(p<0.001)$. There was no difference in length of the aforementioned ligaments $(p \geq 0.056)$.

Conclusion: With this case control study, MRI data on the position of the osseous and ligamentous structures of the cranio-cervical junction in patients with rheumatoid arthritis are available for the first time. Cranialization of the dens axis and signal changes of the transverse ligament and the alar ligaments are the most obvious findings. In a next step, the diagnostic value of MRI of the cervical spine in RA should be prospectively investigated and the available scoring system developed into an internationally established outcome measure.

Disclosure of Interests: [2] Katharina Ziegeler: None declared, Christoph Korsing: None declared, Mattias Bollow: None declared, Kay Geert A. Hermann Speakers bureau: AbbVie, MSD, Pfizer, UCB, Samsung

[3] DOI: 10.1136/annrheumdis-2019-eular.3632

\section{THU0622B PREVENTION OF ACTIVE TUBERCULOSIS INFECTION USING INTERFERON GAMMA RELEASING ASSAY (IGRA) IN PATIENTS WITH RHEUMATOID ARTHRITIS UNDERGOING TUMOR NECROSIS FACTOR-ALPHA INHIBITORS TREATMENT}

Joongyub Lee ${ }^{1}$, Sang Hyun Joo' ${ }^{2}$, Yeong Wook Song ${ }^{2} .{ }^{1}$ Inha University Hospital, Incheon, Korea, Rep. of (South Korea); ${ }^{2}$ Seoul National University, Seoul, Korea, Rep. of (South Korea)

Background: Data on effectiveness reducing incidence of active tuberculosis (TB) by use of interferon gamma releasing assay (IGRA) have not yet been available.

Objectives: To evaluate whether diagnosis of latent tuberculosis infection (LTBI) using IGRA is effective in reducing occurrence of active-TB among patients with RA who will use Tumor Necrosis Factor alpha inhibitors (TNFi).

Methods: A retrospective cohort study was conducted using the National Health Information Database of Korea between 2008 and 2017. The enrollees had at least two claims with the RA diagnosis code, and has at least one prescription of TNFi since 2009 when national insurance of Korea started to cover the expense of IGRA. Exclusion criteria were organ transplantation, HIV infection, or active TB infection within 1 year prior to enrollment. The follow up was initiated on the day of the first prescription for TNFi, and ended on the date of the first claim on active TB, the date of death or December 31, 2017 whichever came first. The utilization of the diagnostic test method was confirmed by the codes for tuberculin skin test (TST) and IGRA claimed within 2 months prior to the initiation of the TNFi. Patients were categorized into 3 groups; no-test, TST only, IGRA (IGRA only or IGRA and TST). The incidence of active TB was defined as the presence of claims with diagnosis code for TB and the start of standard 3-drug or 4-drug first line anti-TB medication combination therapy.

Descriptive statistics on baseline characteristics of participants in each group were presented before and after application of weighting method using standardized mortality rate weight (SMRW). The incidence of active TB in each group was presented as event per 1000 person-year (PY). We treated death as competing event to the occurrence of TB. Therefore a sub-distributional hazard model was used to calculate weighted hazard ratio (wHR) and $95 \%$ confidence intervals $(\mathrm{Cl})$.

Results: Among the 27,021 patients who started TNFi, 15,538 RA patients were included in the analysis through inclusion and exclusion criteria. There were 6,287 patients in the no-test group, 4,677 patients in the TST group, and 4,574 patients in the IGRA group. Diabetes mellitus, chronic kidney disease, and congestive heart failure were more prevalent in IGRA group, while stroke was observed less frequently in IGRA group. After weighting using SMRW, all confounders were well balanced. During the follow up period, 4.24, 4.93, and 4.01 active TB events per 1,000 PY were observed for no-test, TST, and IGRA group, respectively. The death rate was $8.63,9.75$ and 7.96 per 1,000 PY for no-test, TST, and IGRA group, respectively. Considering death as competing event, IGRA significantly reduced the risk of active TB than no-test group (wHR
0.628, 95\% Cl: $0.459-0.859$ ) and TST group (wHR 0.416, 95\% Cl: $0.311-$ 0.556 ).

Conclusion: The incidence of active TB in RA patients using TNFi could be reduced by use of IGRA.

\section{REFERENCES:}

[1] Internal Clinical Guidelines Team (UK). Tuberculosis: Prevention, Diagnosis, Management and Service Organisation. London: National Institute for Health and Care Excellence (UK); 2016 Jan. (NICE Guideline, No. 33.) Available from: https://www.ncbi.nlm.nih.gov/books/NBK338750/

[2] Joint Committee for the Development of Korean Guideline for Tuberculosis, Korean Centers for Disease Control and Prevention. Korean guidelines for tuberculosis. 2nd ed. Seoul: Medrang Inforang Ltd;2014

[3] Jeong DH, Kang J, Jung YJ, Yoo B, Lee CK, Kim YG, Hong S, Shim TS Jo KW. Comparison of latent tuberculosis infection screening strategies before tumor necrosis factor inhibitor treatment in inflammatory arthritis: IGRA-alone versus combination of TST and IGRA. PLoS One. $2018 \mathrm{Ju}$ 5;13(7):e0198756. doi:10.1371/journal.pone.0198756.

Disclosure of Interests: None declared

DOI: 10.1136/annrheumdis-2019-eular.6485

\section{Public health, health services research, and health economics} \section{OF PATIENTS WITH RHEUMATIC DISEASES}

L María. García Vivar ${ }^{1}$, Javier de Toro-Santos ${ }^{2}$, Lucía Pantoja ${ }^{3}$, Cristina Lerín Lozano ${ }^{4}$, Silvia García-Díaz ${ }^{5}$, Sabela Fernández ${ }^{6}$, Yvonne Mestre ${ }^{6}$, Lidia Feo-Lucas ${ }^{6}$, Luis Cea-Calvo ${ }^{6} .{ }^{1}$ Hospital Universitario de Basurto, Bilbao, Spain; ${ }^{2}$ Hospital Universitario A Coruña, A Coruña, Spain; ${ }^{3}$ Hospital del Bierzo, Ponferrada, Spain; ${ }^{4}$ Hospital de Manacor, Manacor, Spain; ${ }^{5}$ Hospital de Sant Joan Despí Moisès Broggi, Barcelona, Spain; ${ }^{6}$ Merck Sharp and Dohme, Madrid, Spain

Background: Non-adherence to medication may lead to poorer clinical outcomes and should be prevented.

Objectives: We describe the frequency of non-adherence behaviors in patients with rheumatic diseases and its relationship to potentially modifiable variables.

Methods: Data were obtained through an anonymous survey handed to patients by physicians or nurses from 25 rheumatology clinics from Spain Patients completed the survey anonymously at home and returned it by pre-paid post mail. Five different non-adherence behaviors were defined. Co-variables analyzed were patients' demographics, medication characteristics, experience with healthcare (assessed with IEXPAC "Instrument to Evaluate the EXperience of PAtients with Chronic diseases" scoring 0 [worst] to 10 [best experience]), and beliefs in medicines (Beliefs About Medicines Questionnaire [BMQ], composed of a necessity and a concerns subscales and scoring -20 [weaker] to +20 [stronger beliefs]). Variables associated to non-adherence were studied with a multivariate logistic regression model.

Results: The survey was handed to 625 patients with rheumatic diseases, of which $336(53.8 \%)$ returned it with the necessary data completed (mean age 55 [14] years, 64\% women). Of them, $188(56.0 \%)$ described at least one non-adherence behavior. The frequencies of the specific non-adherence behaviors were: 1) Forgetfulness in taking medication: $28.6 \%$; 2) Taking medication at unscheduled hours: $5.6 \%$; 3) Stopping medication when feeling well: $10.1 \%$; 4) Stopping medication when feeling sick: $33.0 \%$ and 5) Stopping medication after reading the patients information leaflet: $11.5 \%$. The frequency of at least one non-adherence behavior was similar by age, gender, educational level, working status or by number of medicines. It was slightly higher in patients needing to take their medication 3-4 times a day (63.2\%) versus 1-2 times a day $(52.4 \%, p=0.089)$. IEXPAC scores were similar in patients with or with out non-adherence behaviors (5.5 [2.0] in both groups, $p=0.960)$. BMQ overall score was lower in patients with non-adherence behaviors $(4.3$ versus 6.5 in those without non-adherence behaviors, $p=0.001$ ). The frequency of non-adherence behaviors did not differ by quartiles of IEXPAC score, but it was higher in patients with lower BMQ score (Q1: $59.5 \%$, Q2: $65.9 \%$, Q3: $54.3 \%$, Q4: $52.0 \%$, p-trend $=0.014)$. The multivariate model (table) confirmed a relationship of non-adherence behaviors with lower BMQ beliefs scores.

Conclusion: Non-adherence behaviors are frequent in patients with rheumatic diseases and are mainly associated to patients' beliefs in their medications (assessed with BMQ). This is an important aspect that can 
be addressed by clinical teams to improve adherence and clinical outcomes.

Table 1. Multivariate analysis. Factors associated to non-adherence behaviors of patients with rheumatic diseases.

\begin{tabular}{|l|c|c|}
\hline & OR (95\% CI) & $\begin{array}{c}\text { p- } \\
\text { value }\end{array}$ \\
\hline Age (1-year increment) & $1.00(0.98-1.02)$ & 0.984 \\
\hline Gender (female versus male) & $1.04(0.57-1.90)$ & 0.892 \\
\hline $\begin{array}{l}\text { Need of taking medication 3-4 times per day } \\
\text { (versus 1-2 times per day) }\end{array}$ & $1.40(0.74-2.66)$ & 0.306 \\
\hline Number of different medicines (1-unit increment) & $1.00(0.89-1.14)$ & 0.967 \\
\hline IEXPAC overall score (1-unit increment) & $1.04(0.90-1.21)$ & 0.581 \\
\hline BMQ overall score (1-unit increment) & $0.95(0.91-1.00)$ & 0.052 \\
\hline
\end{tabular}

Acknowledgement:

Funded by Merck Sharp \& Dohme of Spain and endorsed by 4 patients associations: ACCU, CONARTRITIS, SEISIDA, FEDE.

Disclosure of Interests: María L. García Vivar: None declared, Javier de Toro-Santos: None declared, Lucía Pantoja: None declared, Cristina Lerín Lozano: None declared, Silvia García-Díaz: None declared, Sabela Fernández Employee of: MSD, Yvonne Mestre Employee of: MSD, Lidia Feo-Lucas Employee of: MSD, Luis Cea-Calvo Employee of: MSD DOI: 10.1136/annrheumdis-2019-eular.2773

\section{THU0624 UNDERSTANDING ETHNIC DIFFERENCES IN THE UTILIZATION OF NONSTEROIDAL ANTI-INFLAMMATORY DRUGS FOR OSTEOARTHRITIS}

Ernest Vina ${ }^{1}$, Michael Hannon ${ }^{2}$, Jazmin Dagnino ${ }^{1}$, C. Kent Kwoh ${ }^{1} .{ }^{1}$ University of Arizona, Rheumatology, Tucson, United States of America; ${ }^{2}$ Pinney Associates, Pittsburgh, United States of America

Background: The prevalence of arthritis-attributable activity limitation, work limitation and severe pain are significantly higher among Hispanics than among non-Hispanic Whites (NHWs) in the US. While Hispanics are less likely to report regular use of nonsteroidal anti-inflammatory drugs (NSAIDs), reasons for this decreased NSAID use are unknown. It is also unclear whether there are ethnic differences in the use of both over-thecounter (OTC) and prescription NSAIDs.

Objectives: To determine: 1) if there are ethnic differences in the use of OTC and prescription oral NSAIDs for knee/hip osteoarthritis (OA); 2) if there are differences in familiarity with and perceptions of efficacy and risk of NSAIDs between Hispanics and NHWs; and 3) if patient attitudes/ beliefs about NSAIDs mediate observed ethnic differences in the use of NSAIDS for OA

Methods: Participants $\geq 50$ years of age with chronic frequent pain due to knee/hip OA completed structured interviews. Data on sociodemographic characteristics, clinical information, actual use of oral NSAIDs for OA treatment (last 6 months), and familiarity with NSAIDs (3 items, yes/ no response) were collected. Perceptions of efficacy (4 items) and risk (3 items) of NSAIDs were evaluated using five-category ordinal response scale questions. Responses were averaged, with higher values indicating higher perception of efficacy/risk. Fisher's exact or Wilcoxon-Mann-Whitney tests were conducted to determine if knowledge and perceptions about NSAIDs differed by ethnicity. Multivariable logistic regression models were built to determine if ethnic differences in NSAID use were mediated by knowledge and perceptions about the medication.

Results: Among knee/hip OA patients, Hispanics $(n=130)$, in comparison to NHWs $(n=204)$, were younger (mean age 61.8 vs. 65.7) and less likely to have an annual income of $\geq \$ 40 \mathrm{~K}(21.6 \%$ vs. $56.5 \%)$. Hispanics, compared to NHWs, had lower odds of using an OTC NSAID (OR 0.57, $95 \% \mathrm{Cl} 0.36-0.90$ ) but greater odds of using a prescription NSAID (OR 1.66, $95 \% \mathrm{Cl} 1.04-2.64$ ) for OA. Hispanics, compared to NHWs, were also less likely to ever hear about OTC and prescription oral NSAID to treat OA or have a good understanding of either oral NSAID type as a treatment for OA (Table 1). Mean [SD] perceived efficacy of OTC and prescription oral NSAIDs were slightly lower among Hispanics than NHWs (2.91 [0.98] vs. 3.12 [0.88], $\mathrm{p}=0.0565 ; 3.03$ [1.02] vs. 3.34 [0.87], $\mathrm{p}=0.0047$; respectively). Mean [SD] perceived risk of prescription NSAIDs was lower among Hispanics than NHWs (2.44 [1.03] vs. 2.82 [1.01], $\mathrm{p}=0.0012$ ). After adjustment for all familiarity with OTC NSAIDs questions, and perceived efficacy and risk of OTC oral NSAIDs scores, the association between OTC NSAID use and ethnicity was attenuated and no longer significant (OR $1.01,95 \% \mathrm{Cl}$ 0.54-1.89). After adjustment for familiarity with and perceptions of efficacy and risk of prescription NSAIDs, the association between prescription NSAID use and ethnicity remained signifcant (OR 2.62, 95\%Cl 1.51-4.54).

Conclusion: Among patients with knee or hip OA, Hispanics were less likely than NHWs to utilize an OTC oral NSAID as treatment for arthritis. They were also less familiar with the use of NSAIDs for OA treatment and less likely to believe in their efficacy. Patient familiarity and perceptions of OTC oral NSAIDs may mediate ethnic differences in the use of NSAIDs for knee/hip OA.

\begin{tabular}{|c|c|c|c|}
\hline & $\begin{array}{l}\text { Hispanics } \\
(\mathrm{n}=130)\end{array}$ & $\begin{array}{c}\text { NHWs } \\
(n=204)\end{array}$ & $p$-value* \\
\hline \multicolumn{4}{|l|}{ OTC NSAIDs, n (\%) } \\
\hline Heard About It & $100(78.13)$ & $192(95.05)$ & $<.0001$ \\
\hline Family/Friends Received It & $67(52.76)$ & 127 (62.25) & 0.1080 \\
\hline Have Good Understanding & $79(63.20)$ & $168(83.58)$ & $<.0001$ \\
\hline \multicolumn{4}{|l|}{ Prescription NSAIDs, n (\%) } \\
\hline Heard About It & $92(74.80 \%)$ & $173(85.22)$ & 0.0274 \\
\hline Family/Friends Received It & $56(45.16 \%)$ & $107(52.45)$ & 0.2120 \\
\hline Have Good Understanding & $78(62.90 \%)$ & $148(74.00)$ & 0.0462 \\
\hline
\end{tabular}

*Fisher's exact

Disclosure of Interests: Ernest Vina Grant/research support from: Astrazeneca, Consultant for: Astrazeneca, Michael Hannon Employee of: Pinney Associates, Jazmin Dagnino: None declared, C. Kent Kwoh Grant/ research support from: Abbvie, EMD Serono, Consultant for: Astellas, EMD Serono, Thusane, Express Scripts, Novartis

DOI: 10.1136/annrheumdis-2019-eular.4401

\section{THU0625 1 CAN WE IMPROVE INPATIENT REFERRALS TO RHEUMATOLOGY IN A TEACHING HOSPITAL IN THE UK?}

Arani Vivekanantham, Zenab Sarwar Mateen, Roseanna Wheatley, Rachael Myers, Pippa Watson, Jayne Little. Manchester University NHS Foundation Trust, Manchester, United Kingdom

Background: Inpatient referrals to rheumatology vary nationally from paper/electronic methods to phone conversations with the rheumatology registrar. In our Trust, which includes two large teaching hospitals in the UK, rheumatology referrals were hand written, faxed to secretaries and then given to the rheumatology registrar. This method was time consuming and referrals often lacked vital information. The referrer would also not know when the registrar had received the referral. A quality improvement project (QIP) in another UK hospital has shown an electronic referral system to be more efficient and safer for patients [1]

Objectives: This QIP aimed to evaluate the current system for inpatient referrals to two rheumatology departments within one large UK NHS trust and to identify aspects for improvement

Methods: Two hundred and ten inpatient rheumatology referrals received between January 2018 - January 2019 were analysed retrospectively for inclusion of important information such as, patient location and referrer's contact details.

Results: The review of current referrals identified several areas for improvement. The results are summarised in Table 1. The reasons for referrals ranged from swollen, painful joints to new rashes and medication reviews.

Table 1. Inclusion of important items in inpatient rheumatology referrals

\begin{tabular}{lccc}
\hline Item & \multicolumn{3}{c}{$\begin{array}{c}\text { Inclusion of item in referral } \\
\mathbf{n}(\%)\end{array}$} \\
\cline { 2 - 4 } & $\begin{array}{l}\text { Wythenshawe } \\
\text { Hospital } \\
(\mathrm{n}=105)\end{array}$ & $\begin{array}{c}\text { Manchester Royal } \\
\text { Infirmary } \\
(\mathrm{n}=105)\end{array}$ & $\begin{array}{c}\text { Combined } \\
(\mathrm{n}=210)\end{array}$ \\
\hline Date of referral & $78(74)$ & $95(90)$ & $173(82)$ \\
Time of referral & $26(25)$ & $7(7)$ & $33(16)$ \\
Name of referrer & $102(97)$ & $92(88)$ & $194(92)$ \\
Referrer's contact details & $38(36)$ & $13(12)$ & $51(24)$ \\
Patient's name, hospital & $104(99)$ & $102(97)$ & $206(98)$ \\
number & & $101(96)$ & $196(93)$ \\
Patient location & $95(90)$ & $75(75)$ & $137(65)$ \\
Past medical history & $62(59)$ & &
\end{tabular}

A process mapping session was undertaken to identify how the referra system could be improved. An electronic referral system, via the loca electronic patient record (EPR), was created. As well as ensuring that there is an audit trail and referrals are received in a timely manner, referrers are provided with a proforma to direct them to supply the 